\title{
Neural network based adaptive PID controller of nonlinear heat exchanger
}

\begin{abstract}
This research presents the design and simulation of nonlinear adaptive control system on the heating process of shell-and-tube heat exchanger model BDT921. Shell-and-tube heat exchanger is a nonlinear process and change in process dynamics cause instability of the PID controller parameters i.e proportional gain, integral time and derivative time. Thus, the PID controller parameters need to be repeatedly retuned. In this study, neural network approach was introduced to auto-tune the controller parameters. The dynamic data from the BDT921 plant was collected to formulate the mathematical model of the process using MATLAB System Identification Toolbox. NARX model was used to represent the heat exchanger. Neural network was used as adaptive system to the PID controller. The neural network model consists of 4 input variables and 4 output variables. Single hidden layer feed forward neural networks with 20 neurons in hidden layer is the optimum topology of the network. The effectiveness of the controller was evaluated based on the set point tracking only. Simulation result proved that the adaptive PID controller was more effective in tracking the set point with faster settling time and lower or no overshoot respond compared to conventional PID controller.
\end{abstract}

Keyword: Nonlinear process; Neural network; Shell-and-tube heat exchanger; Adaptive controller 